\title{
Measurement Method of Distributed Nodes in Wireless Sensor Networks Based on Multiple Attributes
}

\author{
Bing Zheng and Jing Yang $(\mathbb{D}$ \\ Department of Information Engineering, Hainan Vocational University of Science and Technology, Haikou 571126, China \\ Correspondence should be addressed to Jing Yang; yj13876195734@163.com
}

Received 8 March 2021; Revised 28 March 2021; Accepted 2 April 2021; Published 22 April 2021

Academic Editor: Shah Nazir

Copyright (C) 2021 Bing Zheng and Jing Yang. This is an open access article distributed under the Creative Commons Attribution License, which permits unrestricted use, distribution, and reproduction in any medium, provided the original work is properly cited.

\begin{abstract}
Wireless sensor network (WSN) is usually organized in a particular area for achieving some specific tasks and functions. It has broad application prospects in military, environmental monitoring, disaster relief, and other many commercial areas. Measuring the significance of distributed nodes is the foundation of many applications of WSN. Therefore, this paper studies the distributed node measurement methods and approaches based on multiple attributes in wireless sensor networks to improve the accuracy of distributed node importance measurement. The entropy weight TOPSIS method is used to calculate the importance index of distributed nodes of degree centrality, eigenvector centrality, compactness centrality, betweenness centrality, K-kernel decomposition centrality, and aggregation coefficient; the PR value of each node is given by combining the PageRank algorithm; and then, the parameters $\alpha$ and $\beta$ are introduced to calculate the comprehensive importance measurement results of each node, to finally obtain the importance measurement results of each node dynamically through the improved node deletion method. The experimental results of the research show that the proposed method can accurately measure the importance of distributed nodes in wireless sensor networks, and the accuracy is as high as $98 \%$.
\end{abstract}

\section{Introduction}

Wireless sensor network is a hot research field in the world, which involves highly interdisciplinary and highly integrated knowledge [1]. It integrates sensor technology, embedded computing technology, modern network and wireless communication technology, distributed information processing technology, and so forth. It can monitor, perceive, and collect information of various environments or monitoring objects in real time through various integrated microsensors. The information is sent by the wireless way and transmitted to the user terminal by a self-organizing multihop network [2], so as to realize the connection of the physical world, the computing world, and the human society. Wireless sensor network has a very broad application prospect. It has potential practical value in many important fields, such as military defense, industry and agriculture, urban management, and biomedicine. It has attracted great attention of academic and industrial circles in many countries [3]. It is considered to be one of the technologies that have a great influence on the $21^{\text {st }}$ century. As a new type of information acquisition and processing mode, wireless sensor network (WSN) has randomly distributed nodes integrated with sensors, data processing units, and communication modules [4].

With the help of built-in various sensors, it can measure the thermal, infrared, sonar, radar, and seismic signals in its surrounding environment, so as to detect many networks including temperature, humidity, noise, and light intensity. With the rapid development of Internet technology, wireless communication technology, and electronic technology, wireless sensor network has attracted wide attention in the world [5]. Although the wireless sensor network is similar to the ad hoc network, there are also differences. The wireless sensor network is a network system integrated with monitoring, control, and wireless communication. The number of nodes is larger (thousands or even tens of thousands), and the distribution of nodes is more intensive. Due to 
environmental impact and limited energy, nodes are more prone to failure [6]. Environment interference and node failure are easy to cause the change of network topology. Usually, most sensor nodes are fixed. The processing capacity, storage capacity, and communication capacity of sensor nodes are very limited. In addition, sensor nodes are small in size and usually carry batteries with limited energy. Due to the large number of nodes, low-cost requirements, wide distribution area, and complex deployment area, some areas cannot even be reached by personnel. Therefore, compared with the traditional wireless network, the primary design goal is to provide high-quality service and bandwidth utilization. Wireless sensor network takes data acquisition as the center, and the primary design goal is the efficient use of energy [7], which is also one of the most important differences between the wireless sensor network and traditional network. The typical application of the wireless sensor network is deployed in a specific area to complete the functions of environmental monitoring, target tracking, and positioning. In these applications, sensor nodes transfer the collected data to the server for processing. In order to ensure the validity of data, the importance ranking information of nodes is often essential.

Xiaoyang Sun et al. designed a distributed wireless sensor node monitoring system, which has been successfully applied to the evaluation of underground explosion damage effectiveness; Wang Lu et al. studied the node importance measurement based on the weighted method [8]. First, considering the global information of the network, the characteristic center vector value of each node was calculated, and the weights of the nodes at both ends and the edges formed a weighted network. The local information of weighted network is used to calculate the node importance of weighted network; and Gang $\mathrm{Hu}$ et al. [9] study the calculation of node importance of complex network based on interpretive structure model and apply interpretive structure model to matrix the relationship between nodes of the directed network to obtain the corresponding adjacency matrix and reachable matrix. The reachable matrix is divided by location and level; the matrix is reduced, and leapfrog and self-adaptive are deleted. The hierarchical digraph of the network is obtained, and the network matrix is weighted to simulate evolution, and the identification and division of network region importance and level importance are given. This method is not only suitable for the hierarchical division and identification of the directed network but also suitable for the node ranking calculation of the directed network. In order to solve the limitations of distributed node measurement, based on multiple attributes, a distributed node measurement method based on multiple attributes is studied, which effectively avoids the limitations of single attribute measurement and improves the accuracy of distributed node importance measurement. The entropy weight TOPSIS method is considered for calculating the importance index of distributed nodes of degree centrality, eigenvector centrality, compactness centrality, betweenness centrality, K-kernel decomposition centrality, and aggregation coefficient; the PR value of each node is given by combining the PageRank algorithm; then, the parameters $\alpha$ and $\beta$ are introduced to calculate the comprehensive importance measurement results of each node, to finally obtain the importance measurement results of each node dynamically through the improved node deletion method.

\section{Distributed Node Measurement Method Based on Multiple Attributes in Wireless Sensor Networks}

The following sections briefly show the details of this section.

2.1. Distributed Node Importance Metrics in Wireless Sensor Networks. Let $G=(V, E)$ be a wireless sensor network, where $V=\left\{v_{1}, v_{2}, \ldots, v_{N}\right\}$ is the set of all distributed nodes in the wireless sensor network, $|V|=N ; E=\left\{e_{1}\right.$, $\left.e_{2}, \ldots, e_{m}\right\} \subseteq V \times V$ is the set of edges between distributed nodes, $|E|=m, A=a_{i j}$ of wireless sensor network, where $a_{i j}=1$ means that distributed node $i$ is connected to distributed node $j$; otherwise, $a_{i j}=0$.

Due to the different evaluation criteria of the importance of distributed nodes, the definition of the importance index of distributed nodes is also different. The importance index of distributed nodes is defined as follows:

(1) Degree centrality:

The degree of distributed node $i$ is defined as the number of neighbors of the node, specifically expressed as

$$
K(i)=\sum_{j \in G} a_{i j} .
$$

(2) Eigenvector centrality:

The eigenvector index considers the status and reputation of distributed nodes in wireless sensor networks and considers the reputation of a single node as a linear combination of the reputation of other nodes, so as to obtain a linear equation group. The eigenvector corresponding to the maximum eigenvalue of the system is the importance of each distributed node, which can be specifically expressed as

$$
C_{e}(i)=\lambda^{-1} \sum_{j=1}^{N} a_{i j} e_{j},
$$

where $\lambda$ is the maximum eigenvalue of the adjacency matrix $A$ and $e=\left(e_{1}, e_{2}, \ldots, e_{n}\right)^{T}$ is the eigenvector corresponding to the maximum eigenvalue $\lambda$ of the adjacency matrix $A$.

(3) Closeness centrality:

Compactness is used to measure the ability of distributed nodes in wireless sensor networks to exert influence on other nodes through wireless networks, which can be specifically expressed as

$$
C_{c}(i)=\frac{(N-1)}{\sum_{j=1}^{N} d_{i j}},
$$


where $d$ represents the shortest distance from node $i$ to node $j$.

(4) Betweenness:

Betweenness is used to measure an individual's social status, which means the number of nodes passing through all the shortest paths in wireless sensor networks. It can be expressed as

$$
C_{b}(i)=\sum_{s<t} \frac{\sigma_{s t}^{i}}{\gamma_{s t}}
$$

where $\gamma$ represents the number of shortest paths between node $s$ and node $t$ and $\sigma$ represents the number of shortest paths between node $s$ and node $t$ through node $i$.

(5) Index of location attributes in wireless sensor networks:

The index of location attribute of wireless sensor network is $\mathrm{K}$-shell centrality. K-shell is a distributed node importance ranking index obtained by the K-kernel decomposition method, and the importance of nodes is measured according to the position of nodes in the whole wireless sensor network [10].

(6) Aggregation coefficient:

Aggregation coefficient is the local attribute of the wireless sensor network of distributed nodes, which reflects the frequency of communication between each node in the wireless sensor network. The aggregation coefficient is inversely proportional to the importance of the distributed node. The larger the calculated value, the weaker the importance of the node. The aggregation coefficient of distributed node $i$ is defined as the actual number of edges $E_{i}$ among $\mu_{i}$ neighboring nodes directly connected to the node divided by the total number of possible edges $C_{k_{i}}^{2}$, which can be expressed as

$$
C_{\tau}(i)=\frac{E_{i}}{C_{\mu_{i}}^{2}}=\frac{2 E_{i}}{\mu_{i}\left(\mu_{i}-1\right)} .
$$

\subsection{Node Importance Measurement Method Based on Multiple Attributes and Node Deletion}

2.2.1. Node Importance Measurement Attribute. A variety of distributed node importance measurement attributes, including degree centrality, intermediate centrality, and proximity centrality. Distributed node measurement attribute not only describes the basic characteristics of nodes but also can be easily calculated. Based on the above reasons, the six basic attributes of degree centrality, eigenvector centrality, compactness centrality, dielectric centrality, k-kernel decomposition centrality, and aggregation coefficient are selected as part of the multiple attributes in the node importance measurement method integrating multiple attributes and node deletion. After the measurement attribute is selected, it is necessary to consider the influence of attribute value on distributed node importance [11], whether it is positively correlated or negatively correlated, and then unify the attribute value with the node importance measurement relationship. The calculated values of the five attributes selected in this paper, namely, medium centrality, eigenvector centrality, compactness centrality, intermediate centrality, and k-kernel decomposition centrality, are positively correlated with the importance of nodes; that is, the larger the calculated result, the more important the node [12]. The results of the clustering coefficient attribute are negatively correlated with the importance of nodes. In order to unify the correlation of the selected attributes, the calculation formula of compactness centrality should be adjusted to change it into a positive correlation attribute. Given that the maximum value of compactness centrality is 1 , the adjusted formula is

$$
C_{\tau}^{\prime}(i)=1-C_{\tau}(i)=1-\frac{2 E_{i}}{\mu_{i}\left(\mu_{i}-1\right)}
$$

2.2.2. PageRank Algorithm. PageRank algorithm is a link analysis algorithm. The idea of the algorithm is that when a high-quality distributed node points to another node, the quality of the other node is related to the quality of the node pointing to it. If the node pointing to it is of good quality and the node being pointed to is of good quality, the regression relationship is used to calculate the importance of the node [13]. The principle of the PageRank algorithm is as follows: firstly, PageRank assigns the same PR value to all nodes in the wireless sensor network; then, it updates the PR value of each node in real time through continuous iterative recursive operation, and so on until the $\mathrm{PR}$ value is stable. The result calculated by PageRank is the result of node importance measurement. Its expression is as follows:

$$
\operatorname{PR}(P)=\frac{1-q}{n}+q \sum_{i=1}^{n} \frac{\operatorname{PR}\left(U_{i}\right)}{N_{U_{i}}},
$$

where $\operatorname{PR}(P)$ is the value $\mathrm{PR}$ of node $P$ and $q$ is the damping factor bounded by the interval $(0,1)$ and represents the probability that the user continues to visit backward in the process of accessing a certain node at any time [14]. The general value is $q=0.85 ; n$ is the total number of nodes; $U_{1}, U_{2}, \ldots, U_{n}$ is the node pointing to node $P$; $P R(U)$ is the $\mathrm{PR}$ value of node $\mathrm{U}$ pointing to node $P$; and $N$ is the number of nodes $U$ points to. According to the calculation formula of PageRank, the importance of $P$ node is related to the amount of data transmission of this node. The more the amount of data transmission is [15], the more important $P$ is. At the same time, the more important the node pointing to node $P$ is, the more important node $P$ is, and the greater the $\mathrm{PR}$ value of $P$ is.

2.2.3. TOPSIS Algorithm Based on Entropy Weight. TOPSIS is a multiobjective decision-making method. The core idea of this method is to first select a positive ideal solution and a negative ideal solution in wireless sensor networks and then take the solution closest to the positive ideal solution and farthest from the negative ideal solution as 
the optimal solution [16]. However, TOPSIS does not explain the rationality of attribute weight setting, so the calculation results are subjective. As an objective weighting method, the entropy weight method is often used to determine the weight of each attribute. Its basic idea is to give weight to each attribute according to the amount of information reflected by the variation degree of each evaluation attribute [17]. Therefore, the concept of "information entropy" is introduced, and the entropy weight method is used in the process of giving multiple attribute weights in TOPSIS; the specific algorithm is as follows:

In the multiattribute measurement problem, the metric matrix $Y=\left\{y_{i q}\right\}$ and the normalized metric matrix $Z=\left\{z_{i q}\right\}$ are set, and the normalized metric matrix is calculated by the vector specification method. The calculation formula is as follows:

$$
z_{i q}=\frac{y_{i q}}{\sum_{i=1}^{n} y_{i q}}, \quad i=1,2, \ldots, n ; q=1,2, \ldots, p,
$$

where $n$ represents the number of distributed nodes; $p$ represents the number of attribute types that measure the importance of nodes; $y_{i q}$ is the value of the $i$-th node on the $g$-th attribute index.

The entropy weight method is used to deal with the canonical metric matrix of multiple attributes, and the weight of each attribute $w_{q}$ is obtained. The entropy weight of the $g$ attribute is expressed as

$$
w_{q}=\frac{1-H_{q}}{p-\sum_{q=1}^{p} H_{q}}, \quad q=1,2, \ldots, p,
$$

where $H_{q}$ represents the information entropy value of the attribute of item $q$, and its expression is as follows:

$$
H_{q}=-k \sum_{i=1}^{n} z_{i q} \ln z_{i q}, \quad i=1,2, \ldots, n ; q=1,2, \ldots, p .
$$

Set the weight of each attribute as $w=\left(w_{1}\right.$, $\left.w_{2}, \ldots, w_{p}\right)^{T}$, and use formula (11) to form a weighted canonical matrix $X=\left\{x_{i q}\right\}$ :

$$
x_{i q}=w_{q} \cdot z_{i q}
$$

Using formulae (12) and (13), the positive ideal solution $x^{*}$ and negative ideal solution $x^{0}$ with each attribute are obtained. Let the $q$-th attribute value of ideal $x^{*}$ be $x_{q}^{*}$. The $q$ th attribute value of the negative ideal solution $x^{o}$ is $x_{q}^{o}$; then,

$$
\begin{gathered}
x_{q}^{*}=\left\{\begin{array}{c}
\max x_{i q} \\
i \\
\max x_{i q} \\
i
\end{array}\right. \\
x_{q}^{o}=\left\{\begin{array}{c}
\max x_{i q} \\
i \\
\max x_{i q} \\
i
\end{array}\right.
\end{gathered}
$$

$d_{i}^{*}$ is the distance between $x_{i}$ and the positive ideal solution, and $d_{i}^{o}$ is the distance between $x_{i}$ and the negative ideal solution. Calculate the distance from each attribute to the positive and negative ideal solutions, respectively, according to equations (14) and (15):

$$
\begin{aligned}
& d_{i}^{*}=\sqrt{\sum_{q=1}^{p}\left(x_{i q}-x_{q}^{*}\right)^{2}}, \quad i=1,2, \ldots, n, \\
& d_{i}^{o}=\sqrt{\sum_{q=1}^{p}\left(x_{i q}-x_{q}^{o}\right)^{2},} \quad i=1,2, \ldots, n .
\end{aligned}
$$

According to formula (16), the comprehensive measurement value of each attribute is calculated, and the value is sorted from large to small, and finally, the importance ranking measurement result of nodes is obtained; the formula is

$$
C_{i}^{o}=\frac{d_{i}^{o}}{d_{i}^{o}+d_{i}^{*}}, \quad i=1,2, \ldots, n .
$$

\subsubsection{Comprehensive Ranking Measurement Method.} Degree centrality, eigenvector centrality, compactness centrality, betweenness centrality, K-kernel decomposition centrality, and aggregation coefficient are six kinds of centrality indexes that affect the ranking of distributed nodes [17]. Starting from the structural characteristics of wireless sensor networks, the importance of nodes is quantitatively analyzed; the PageRank algorithm theory is used to accurately locate nodes in wireless sensor networks importance, on the basis of the above, through the improved node removal method to investigate the influence of the change of wireless sensor network connectivity on the importance of nodes. Based on the above three main research methods of node importance in wireless sensor networks, a node importance measurement method combining multiple attributes and node deletion is proposed; the specific process of this method is shown in Figure 1.

Figure 1 shows the entropy-based multiattribute method and the node types in distributed wireless sensor networks. It is assumed that the node importance metric based on the PageRank algorithm is calculated as $\operatorname{PR}(i)$, introducing $\alpha, \beta$ to $C_{i}^{o}$ and get linear regression node scheduling $\mathrm{PR}(i)$ measurement $C_{i}$, node deletion method based on the improved dynamic get distributed node importance ranking of four parts; the concrete steps of the algorithm are as follows:

Step 1: according to the acquired about the connection between the node and the node data to establish the corresponding wireless sensor network, computing degree of each node in wireless sensor network centricity, characteristic vector centricity, tightness of centricity, betweenness centrality, $K$ nuclear decomposition of centricity, and six class attribute values and then by formula (8), the calculation results being normalized processing, establish a standardized measure of $n \times 4$ matrix node properties.

Step 2: the concept of information entropy is introduced into the determination of weight, and the normalized metric matrix obtained in step 1 is calculated 


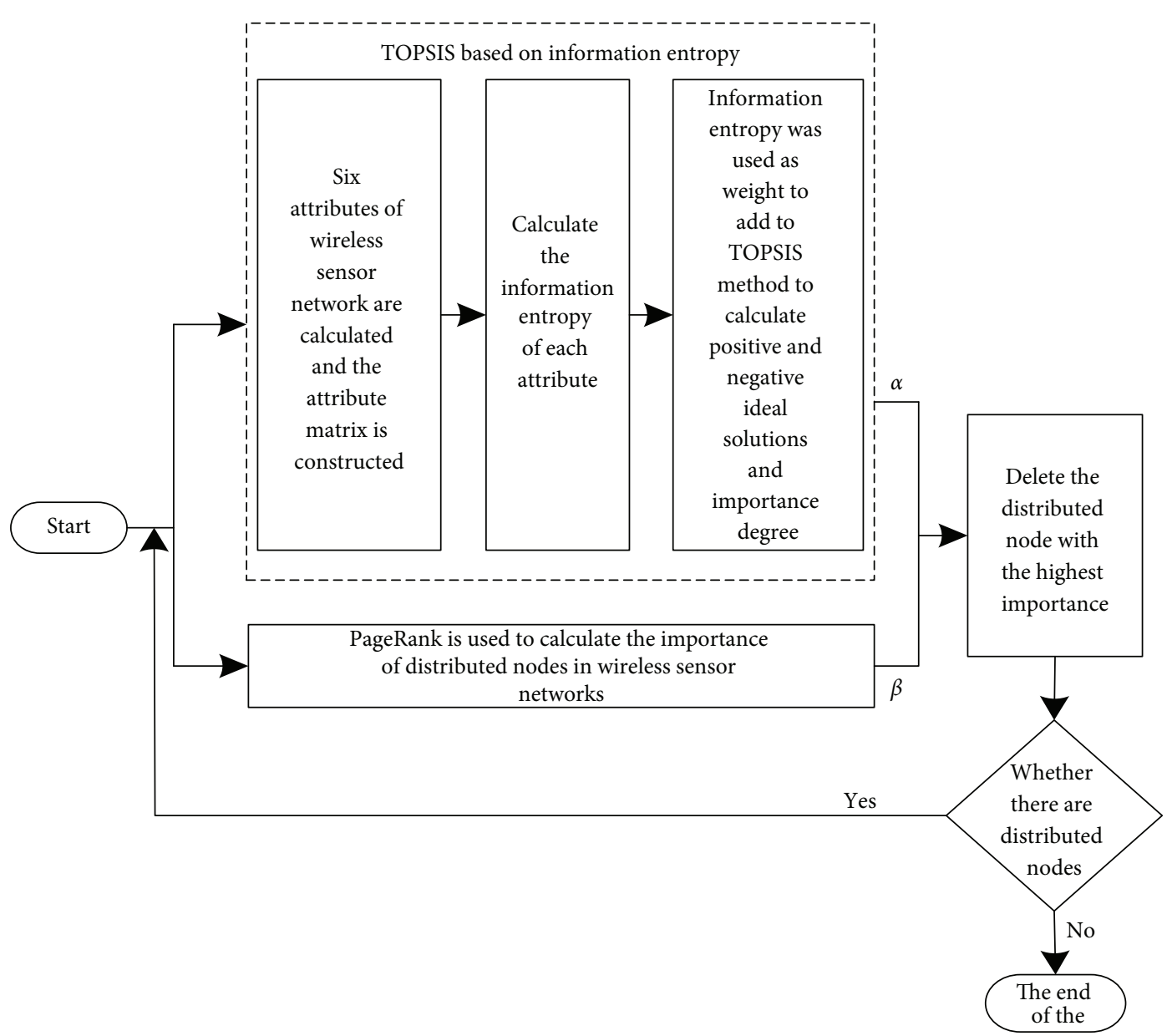

FIGURE 1: Flow chart of node importance measurement method integrating multiple attributes and node deletion.

by using the entropy weight method to obtain the entropy weights corresponding to various attributes [18], and then the weighted canonical matrix is obtained.

Step 3: the six kinds of attribute values of distributed nodes are taken as multiple attributes in the TOPSIS method, the entropy weight corresponding to each attribute is taken as the attribute weight in the TOPSIS method, and the positive and negative ideal solutions of each attribute are calculated.

Step 4: obtain the importance ranking $C_{i}^{o}$ of distributed nodes.

Step 5: the PageRank algorithm is used for reference and its principle is introduced to calculate the $P R$ value of distributed nodes PR(i). According to the PR value, the importance measurement results of nodes can be obtained and taken as a part of the importance measurement of wireless sensor network nodes.

Step 6: normalize the PR value of distributed nodes. Linear regression is conducted between the node ranking measurement result $C_{i}^{o}$ calculated based on entropy weight TOPSIS method and the node ranking measurement value PR calculated based on PageRank algorithm to obtain the improved node importance comprehensive ranking measurement result $C_{i}$. The specific calculation formula is as follows:

$$
C_{i}=\alpha \cdot C_{i}^{o}+\beta \cdot \operatorname{PR}(i)
$$

$\alpha$ and $\beta$ represent the weighting coefficients of node importance of TOPSIS method and PageRank node importance based on multiattribute entropy weight, respectively, $\alpha+\beta=1$.

Step 7: on the basis of the above steps, first disconnect the $C_{i}$ value maximum distributed nodes in wireless sensor network connection. Then connect the disconnected node to the node whose PR value is evenly distributed, and then according to the steps one to six to, recalculate the importance of remaining in the new wireless sensor network node measurement [19, 20], then disconnect the new $C_{i}$ value maximum in the wireless sensor network node, and recalculate the importance of the residual node values. This cycle continues until only the last node is left in the wireless sensor network, thus obtaining the final node importance ranking metric. 


\section{Experimental Analysis}

In order to verify the effectiveness and relative accuracy of this method, the simulation experiment of wireless sensor network distributed node measurement is carried out in MATLAB, and 20 and 100 randomly distributed nodes are set in the simulation program. The topology of the wireless sensor network in two cases is shown in Figure 2.

3.1. Effectiveness Analysis. Taking a wireless sensor network with 20 distributed nodes as an example, six kinds of attribute values of each distributed node in the wireless sensor network are calculated through simulation experiments, including degree centrality, eigenvector centrality, compactness centrality, betweenness centrality, K-kernel decomposition centrality, and aggregation coefficient. The calculation results of each attribute value are shown in Table 1; the result of the calculation is rounded to four decimal places.

Then, the normalization decision matrix is obtained by using the vector norm method, the entropy weight method is used to process the decision matrix, the information entropy value $H_{q}$ and weight $w_{q}$ of each attribute are obtained, and the result retains four decimal numbers where the information entropy values of each attribute are, respectively, $H_{1}=0.9979$, $H_{2}=0.9429, H_{3}=0.9987, H_{4}=0.9939, H_{5}=0.9915$, and $H_{6}=0.9865$. The attribute weights are, respectively, $w_{1}=0.1412, \quad w_{2}=0.6863, \quad w_{3}=0.2495, \quad w_{4}=0.1819$, $w_{5}=0.1607$, and $w_{6}=0.5641$. Then, the weighted gauge matrix $X$ is obtained. After that, the positive ideal solution $x^{*}$ and negative ideal solution $x^{0}$ for each attribute are calculated, and the calculated result remains four decimal places, positive ideal solution $x^{*}=(0.0111,0.0793,0.0015,0.0089)$, and negative ideal solution $x^{o}=(0.0051,0,0.0010,0)$. Finally, the distance between each attribute in the wireless sensor network and the positive ideal solution and the distance between each attribute and the negative ideal solution are calculated, and the comprehensive measurement value of each attribute is calculated. Finally, the distributed node importance value $C_{i}^{o}$ of TOPSIS method based on the entropy weight of multiple attributes is obtained. The importance value and ranking measurement results of each node are shown in Table 2.

PageRank algorithm is used to calculate the PR value of each node in the wireless sensor network. The node PR value and ranking measurement results of the PageRank algorithm are shown in Table 3.

It can be seen from Tables 2 and 3 that the ranking of distributed nodes obtained by evaluating the importance of distributed nodes from different perspectives is not identical. In order to more comprehensively and more accurately get the importance of distributed node measurement, in the calculation method of TOPSIS attribute based on entropy, this method recalculates the importance ranking index of distributed nodes and the nodes after the PR value. Among them, the normalization processing and PR value are performed based on the Page Rank algorithm, and the proportion is introduced. The coefficients $\alpha$ and $\beta$ are postprocessing linear regression calculated values. The results accord with the actual circumstances of the wireless sensor network and distributed nodes. The proportionality coefficient is $\alpha=0.7, \beta=0.3$, after determining the proportionality coefficient; the comprehensive ranking measurement result of node importance of wireless sensor network containing 20 distributed nodes is calculated.

Then, the node ranked first in the measurement result is deleted, and its PR value is evenly distributed to the neighbor nodes connected with it, making it a part of the PR value of neighbor nodes. Then, the remaining deleted nodes were used as a new wireless sensor network. The above steps were repeated to recalculate the attribute value, attribute weight, $C_{i}^{o}, \mathrm{PR}$ value, and comprehensive ranking $C_{i}$ of the remaining nodes to obtain the new distributed node ranking measurement result. Then, continue to delete the first ranked node, until there is only one node left in the wireless sensor network. The importance of each distributed node in the wireless sensor network is measured by the method in this paper, and the ranking result of the final measurement is as follows $[1-19,21,22]$.

Network efficiency indicates the connectivity of the wireless sensor network. The higher the efficiency of the wireless sensor network is, the better the connectivity is. According to different importance metrics of distributed nodes, the same proportion of distributed nodes are deleted, and the larger the decline rate of network efficiency is, which indicates that the greater the impact of these distributed nodes on network efficiency is, the better the attack effect is, and the corresponding distributed nodes are selected. The point importance measure is more effective. In order to compare and verify the effectiveness of this method, which uses multiple attributes as metrics to measure the importance of distributed nodes in wireless sensor networks, six single attribute metrics, namely, degree centrality, eigenvector centrality, compactness centrality, betweenness centrality, $K$ -kernel decomposition centrality, and aggregation coefficient, and multiple attribute metrics are used to measure the importance of distributed nodes in two wireless sensor networks. In this case, the network efficiency after the deletion of distributed nodes is compared and analyzed. The deletion of distributed nodes in the wireless sensor network is mainly to delete the top $10 \%$ sorted according to different metrics, and the proportion of deleted distributed nodes is from $0 \%$ to $20 \%$. At the same time, the efficiency decline proportion of the wireless sensor network after the deletion of distributed nodes is calculated. The experimental results are shown in Figure 3.

According to the analysis of Figure 3, with the gradual increase of the proportion of deleted distributed nodes, the decline proportion of network efficiency using single attribute measurement index and multiple attribute measurement index in the two sizes of wireless sensor networks is increasing, and the decline proportion trend of network efficiency of the two types of wireless sensor networks is basically the same; for wireless sensor networks with fewer distributed nodes, the decline proportion of multiple attribute measurement index is increasing. When the proportion of deleting distributed nodes is less than $10 \%$ in the wireless sensor network with more distributed nodes, the gap between single attribute metrics and multiple attribute metrics is not obvious, but the overall network efficiency decline proportion of multiple attribute metrics is low. For each single attribute metric, when the 


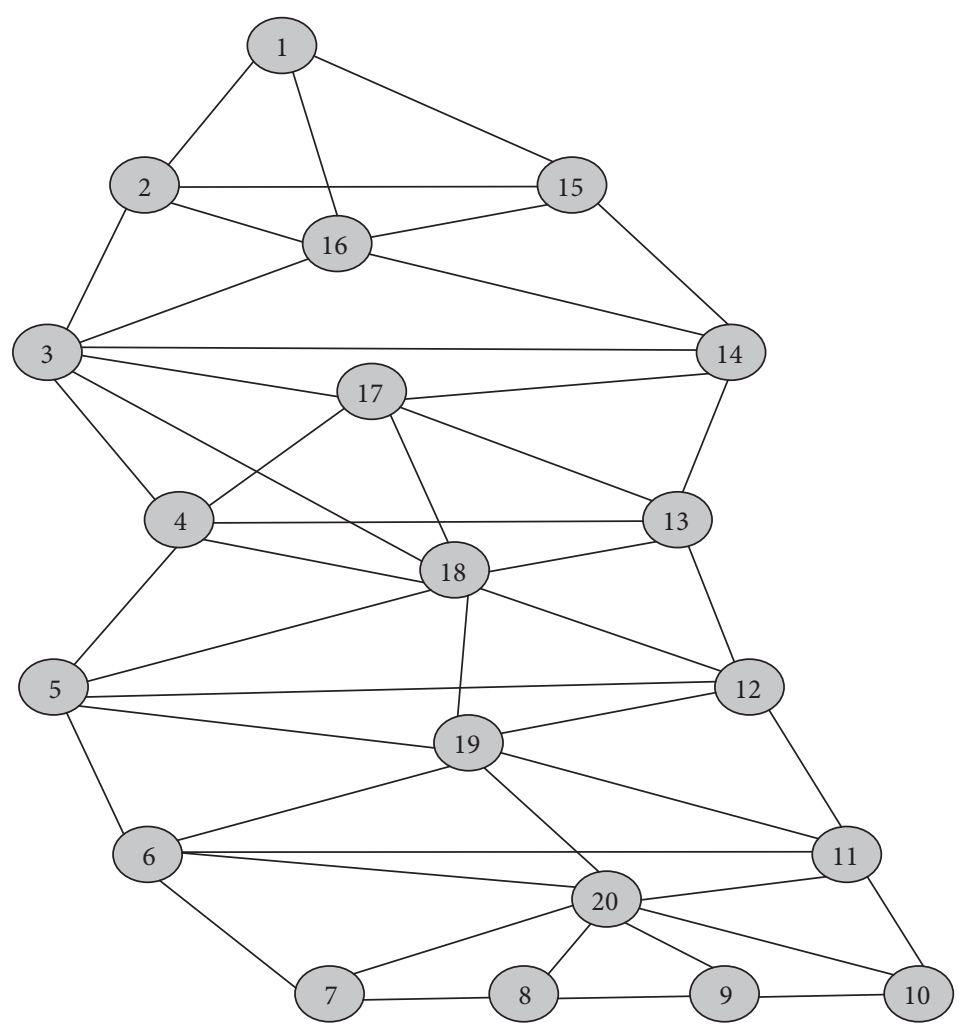

(a)

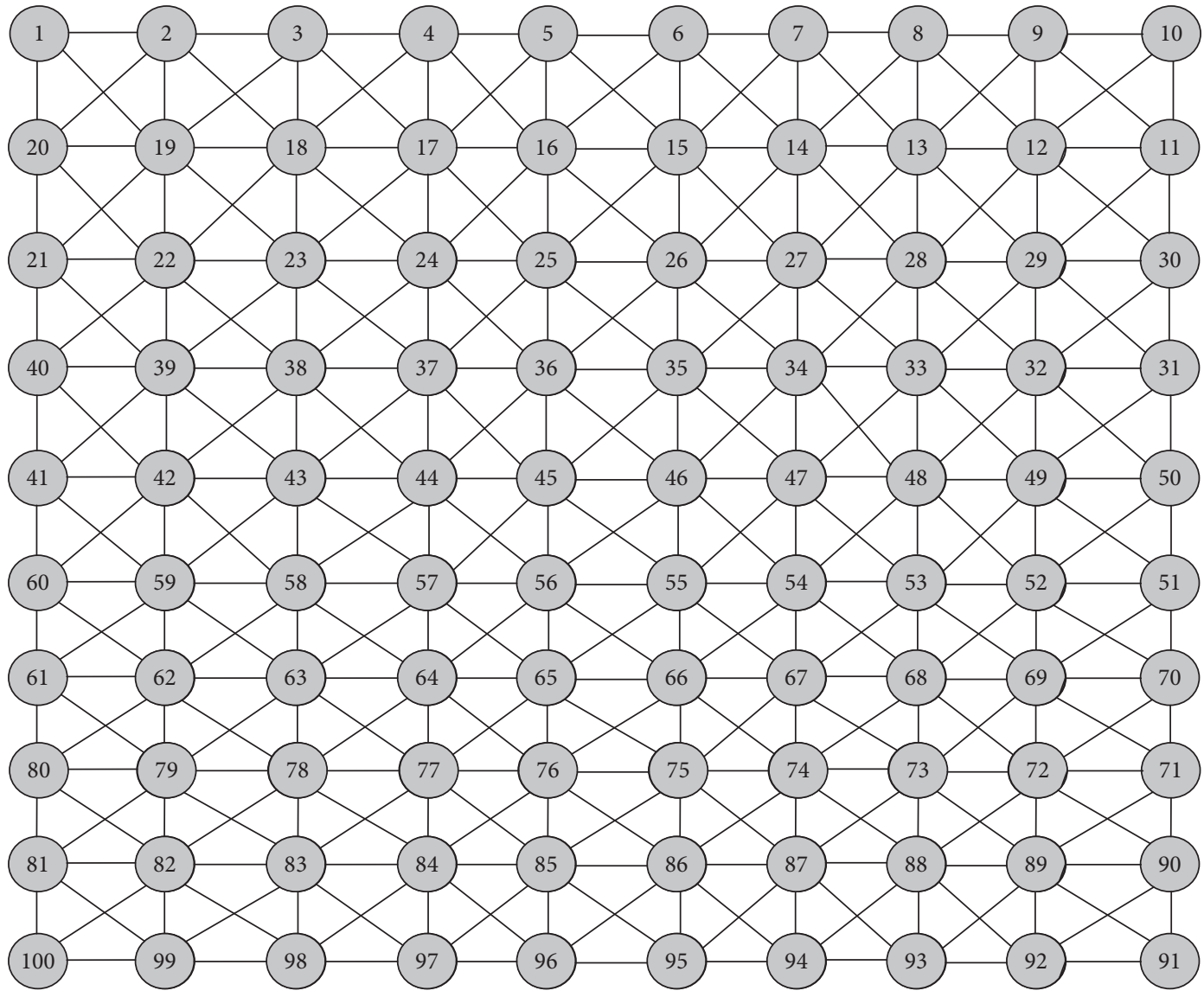

(b)

Figure 2: Topological structure of wireless sensor network. (a) Wireless sensor network topology with 20 distributed nodes. (b) Wireless sensor network topology with 100 distributed nodes. 
TABLE 1: Attribute values of each distributed node.

\begin{tabular}{|c|c|c|c|c|c|c|}
\hline $\begin{array}{l}\text { Distributed } \\
\text { node }\end{array}$ & $\begin{array}{l}\text { Degree of } \\
\text { centricity }\end{array}$ & $\begin{array}{l}\text { Centrality of } \\
\text { eigenvectors }\end{array}$ & $\begin{array}{c}\text { Compactness } \\
\text { centrality }\end{array}$ & $\begin{array}{c}\text { Intermediate } \\
\text { centrality }\end{array}$ & $\begin{array}{c}\text { K nuclear decomposition } \\
\text { centrality }\end{array}$ & $\begin{array}{c}\text { Aggregation } \\
\text { coefficient }\end{array}$ \\
\hline$\overline{1}$ & 0.1111 & 0.1532 & 0.2643 & 0.0000 & 0.1310 & 0.0000 \\
\hline 2 & 0.2111 & 0.1885 & 0.2996 & 0.1592 & 0.1663 & 0.8444 \\
\hline 3 & 0.2111 & 0.2571 & 0.3682 & 0.3269 & 0.2349 & 1.1111 \\
\hline 4 & 0.1111 & 0.3175 & 0.3286 & 0.1895 & 0.2953 & 1.1111 \\
\hline 5 & 0.1111 & 0.1843 & 0.2952 & 0.1677 & 0.1621 & 1.1111 \\
\hline 6 & 0.1611 & 0.1843 & 0.2952 & 0.2124 & 0.1621 & 1.1111 \\
\hline 7 & 0.1111 & 0.1532 & 0.2643 & 0.1177 & 0.1310 & 1.1111 \\
\hline 8 & 0.1111 & 0.1288 & 0.2399 & 0.0699 & 0.1066 & 1.1111 \\
\hline 9 & 0.1111 & 0.1326 & 0.2437 & 0.0729 & 0.1104 & 1.1111 \\
\hline 10 & 0.1111 & 0.1532 & 0.2643 & 0.1186 & 0.1310 & 1.1111 \\
\hline 11 & 0.1111 & 0.1857 & 0.2968 & 0.1668 & 0.1635 & 1.1111 \\
\hline 12 & 0.1611 & 0.2380 & 0.3491 & 0.2817 & 0.2158 & 1.1111 \\
\hline 13 & 0.1111 & 0.2175 & 0.3286 & 0.1699 & 0.1953 & 1.1111 \\
\hline 14 & 0.2111 & 0.2175 & 0.3286 & 0.1857 & 0.1953 & 1.1111 \\
\hline 15 & 0.1611 & 0.1778 & 0.2889 & 0.0572 & 0.1556 & 0.6778 \\
\hline 16 & 0.1111 & 0.1740 & 0.2851 & 0.0229 & 0.1518 & 1.1111 \\
\hline 17 & 0.1111 & 0.2077 & 0.3188 & 0.0585 & 0.1855 & 1.1111 \\
\hline 18 & 0.1111 & 0.2333 & 0.3444 & 0.1298 & 0.2111 & 1.1111 \\
\hline 19 & 0.1611 & 0.2448 & 0.3559 & 0.2265 & 0.2226 & 1.1111 \\
\hline 20 & 0.1111 & 0.1885 & 0.2996 & 0.0985 & 0.1663 & 1.1111 \\
\hline
\end{tabular}

TABLE 2: Importance values of each node and ranking measurement results.

\begin{tabular}{lc}
\hline Distributed node & The importance of value \\
\hline 3 & 1.1000 \\
12 & 0.8661 \\
19 & 0.6951 \\
6 & 0.6515 \\
4 & 0.2512 \\
14 & 0.5694 \\
13 & 0.5179 \\
5 & 0.5112 \\
11 & 0.4985 \\
2 & 0.4891 \\
18 & 0.3989 \\
10 & 0.3619 \\
7 & 0.3593 \\
20 & 0.3319 \\
9 & 0.2578 \\
8 & 0.2492 \\
17 & 0.1912 \\
15 & 0.1744 \\
16 & 0.1192 \\
1 & 0.0012 \\
\hline
\end{tabular}

proportion of deleted distributed nodes is more than $10 \%$, the network efficiency decline proportion of multiattribute metric is significantly higher than that of single attribute metric; comprehensive analysis shows that the network efficiency decline proportion of multiattribute metric is the smallest. Experiments show that this method uses multiple attributes as the distributed node importance measurement index; compared with each single attribute measurement index, it has a better measurement effect on the wireless sensor network. Through the comparative analysis of the network efficiency decline ratio after the deletion of distributed nodes, the multiple attribute measurement index can better measure the importance of distributed nodes.
TABLE 3: Node PR value and ranking measurement results.

\begin{tabular}{lc}
\hline Distributed node & PR value \\
\hline 3 & 0.0711 \\
14 & 0.0698 \\
2 & 0.0682 \\
6 & 0.0619 \\
12 & 0.0585 \\
19 & 0.0582 \\
15 & 0.0524 \\
9 & 0.0459 \\
8 & 0.0457 \\
10 & 0.0452 \\
7 & 0.0445 \\
11 & 0.0433 \\
5 & 0.0429 \\
20 & 0.0423 \\
4 & 0.0422 \\
18 & 0.0396 \\
13 & 0.0393 \\
17 & 0.0377 \\
16 & 0.0371 \\
\hline
\end{tabular}

3.2. Comparative Analysis. In this paper, the node importance measurement method based on the weighted method (reference [8]) and the node importance measurement method of complex network based on interpretive structure model (reference [9]) are used to measure the importance of distributed nodes in wireless sensor networks. The two methods are recorded as method 1 and method 2. As a comparison method of the methods in this paper, the performance of the three methods under different network connectivity is tested. The test results are shown in Figure 4 .

It can be seen from the analysis of Figure 4 that in twoscale wireless sensor networks, the measurement accuracy of the three methods improves with the increase of network 


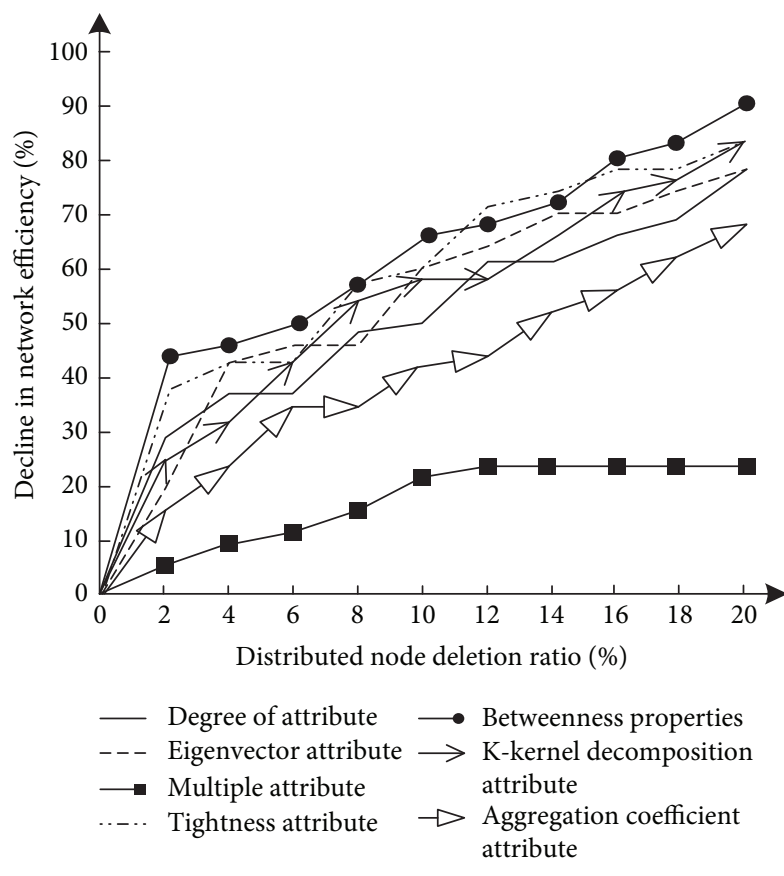

(a)

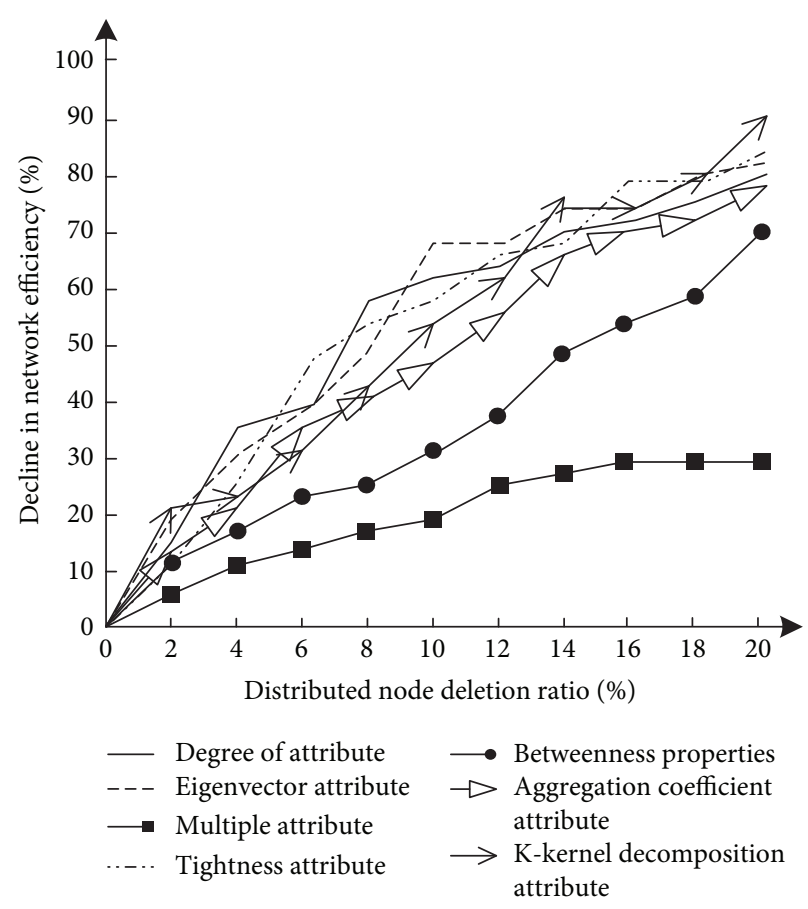

(b)

FIGURE 3: Ratio of efficiency decline of the wireless sensor network. (a) Decline ratio of network efficiency of wireless sensor network with 20 distributed nodes. (b) Decline ratio of network efficiency of wireless sensor network with 100 distributed nodes.

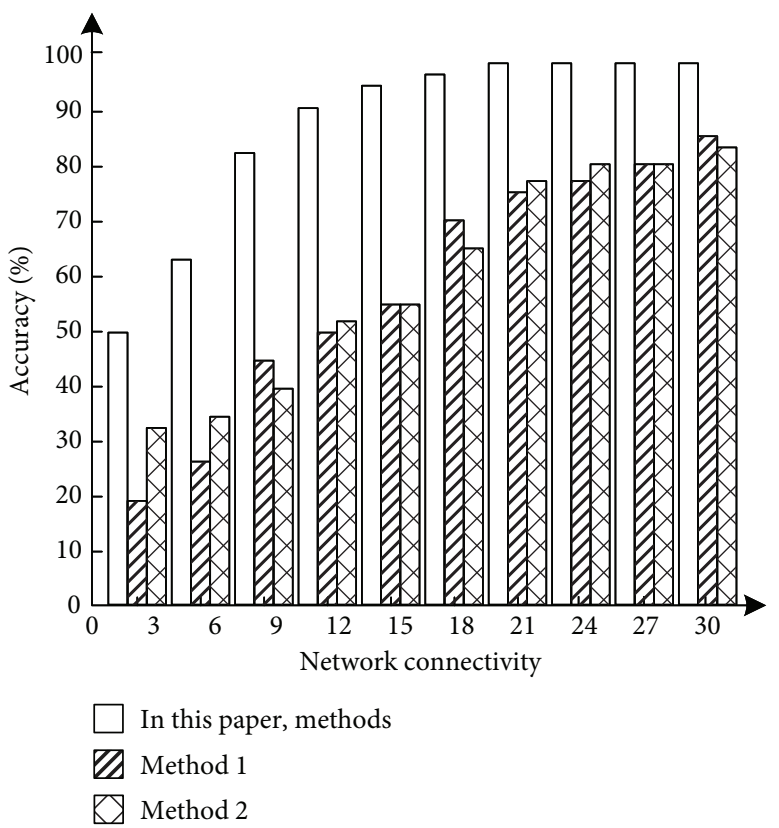

(a)

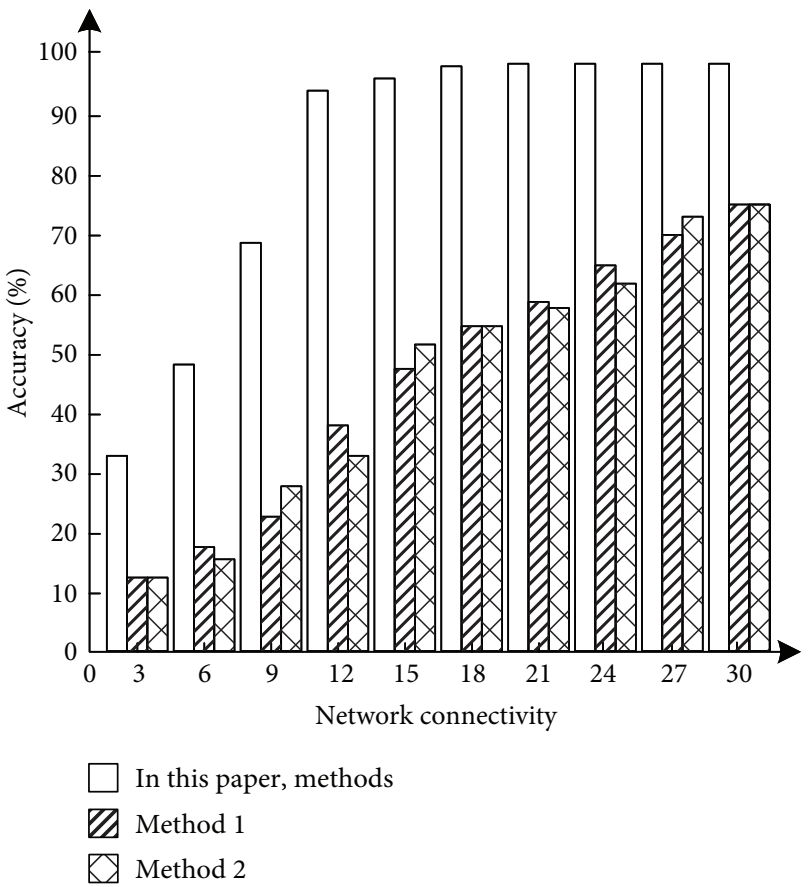

(b)

Figure 4: Three methods of the distributed node measurement accuracy. (a) 20 distributed nodes of wireless sensor network distributed node measurement accuracy. (b) 100 distributed nodes of wireless sensor network distributed node measurement accuracy.

connectivity. In two-scale wireless sensor networks, the measurement accuracy of this method is higher than that of the other two methods. In larger scale wireless sensor networks, the measurement accuracy of this method is higher than that of the other two methods. When the network connectivity reaches a certain degree, the 
measurement accuracy of this method tends to be stable. In the small-scale wireless sensor network, the measurement accuracy of this method is as high as $96 \%$, and the measurement accuracy of the other two methods is not as high as $90 \%$; in the large-scale wireless sensor network, the measurement accuracy of this method is as high as $98 \%$, and the measurement accuracy of the other two methods is not as high as $90 \%$. The accuracy of the method is about $75 \%$. Experimental results show that the proposed method has the highest accuracy in measuring the importance of distributed nodes in wireless sensor networks.

\section{Conclusion}

Wireless sensor network integrates sensor technology, microelectronics technology, wireless communication, and network technology. WSN has broad application prospects in the fields of data acquisition, environmental monitoring, target tracking, and many other areas. However, due to the characteristics of self-organization, large number of nodes, limited resources, and constantly changing network topology, node measurement has become a crucial problem to be solved. At present, a variety of measurement methods have been proposed, but these methods often have the disadvantages of low measurement accuracy, high complexity, and weak fault tolerance. In view of the existing importance ranking methods of distributed nodes in wireless sensor networks, such as the one sidedness of evaluation index, the objectivity of attribute weight setting, the neglect of network structure changes, and the influence of important neighbor nodes, a distributed node importance measurement method based on multiple attributes for WSN is proposed. This method uses various attributes to effectively and efficiently avoid the limitations of single attribute measurement; introduces the concept of information entropy into TOPSIS algorithm to weight the multiple attributes of nodes; eliminates the error caused by artificial weight; and uses page for reference. Rank algorithm is improved to make it a part of the importance measurement of distributed nodes; the improved node deletion method and repeated calculation are used to comprehensively measure the importance of nodes, so as to reduce the influence of topology changes and important neighbor nodes and improve the accuracy of the importance measurement of distributed nodes. The experimental results achieved the effectiveness of the proposed research.

\section{Data Availability}

The data used to support the findings of this study are available from the corresponding author upon request.

\section{Conflicts of Interest}

The authors declare that there are no conflicts of interest regarding the publication of this paper.

\section{Acknowledgments}

The authors acknowledge Beichuang teaching assistant fund, University Industry Research Innovation Fund of Science and Technology Development Center of Ministry of Education (Grant no. 2018A05031).

\section{References}

[1] Y. Guangyou, X. Chenbo, G. Xiong, and Z. Tuo, "Energy efficient node deployment optimization for cts based on cooperative beamforming in single-hop wireless sensor networks," Automatic Control and Computer Sciences, vol. 54, no. 2, pp. 147-155, 2020.

[2] D. Peng, J. Zhongliang, S. Kai, and L. Minzhe, "A distributed consensus filter for sensor networks with heavy-tailed measurement noise. Sciece China," Information Sciences, vol. 61, no. 11, Article ID 119201, 2018.

[3] S. Dolha, P. Negirla, F. Alexa, and I. Silea, "Considerations about the signal level measurement in wireless sensor networks for node position estimation," Sensors, vol. 19, no. 19, pp. 41-79, 2019.

[4] M. Zhang, X. Zhang, and Y. Huang, "Data collection optimization method for wireless sensor networks based on linear regression," IOP Conference Series Materials Science and Engineering, vol. 569, Article ID 032064, 2019.

[5] E. H. Houssein, M. R. Saad, K. Hussain, W. Zhu, H. Shaban, and M. Hassaballah, "Optimal sink node placement in large scale wireless sensor networks based on harris' hawk optimization algorithm," IEEE Access, vol. 8, no. 99, pp. 19381-19397, 2020.

[6] S. B. Chandanapalli, E. S. Reddy, and D. R. Lakshmi, "Dftdt: distributed functional tangent decision tree for aqua status prediction in wireless sensor networks," International Journal of Machine Learning and Cybernetics, vol. 9, no. 9, pp. 1419-1434, 2018.

[7] W. Xuqi, H. Fangyuan, G. Pengfei, and W. Wenqing, "Localization model of wireless sensor network node integrated with constrained convergence distance," IPPTA: Quarterly Journal of Indian Pulp and Paper Technical Association, vol. 30, no. 8, pp. 840-845, 2018.

[8] L. Wang, Q. Guo, and J. G. Liu, "Measuring node importance based on weighted nonlinear method," Application Research of Computers, vol. 35, no. 5, pp. 1426-1428, 2018.

[9] G. Hu, X. X. Xu, and X. C. Guo, "Importance calculation of complex network nodes based on interpretive structural modeling method," Journal of Zhejiang University(Engineering Science), vol. 52, no. 10, pp. 1989-1997+2022, 2018.

[10] S. S. R. Krishnan and A. Thangavelu, "An early prevention method for node failure in wireless sensor networks," International Journal of Internet Technology and Secured Transactions, vol. 10, no. 5, p. 507, 2020.

[11] M. A. Jan, P. Nanda, X. He, and R. P. Liu, "A Sybil attack detection scheme for a forest wildfire monitoring application," Future Generation Computer Systems, vol. 80, pp. 613-626, 2018.

[12] Y.-R. Lee, X. Jin, and H.-N. Kim, "Node localization based on neural network using semi-supervised learning in wireless sensor networks," The Journal of Korean Institute of Communications and Information Sciences, vol. 44, no. 3, pp. 517-527, 2019.

[13] Y. Huang, H. Wang, and K. Li, "An anchor node selection mechanism-based node localisation for mines using wireless sensor networks," International Journal of Computational Science and Engineering, vol. 1, no. 1, p. 1, 2019.

[14] M. A. Jan, M. Usman, X. He, and A. U. Rehman, "SAMS: a seamless and authorized multimedia streaming framework for 
WMSN-based IoMT," IEEE Internet of Things Journal, vol. 6, no. 2, pp. 1576-1583, 2018.

[15] Z. J. Jiang, T. Zhou, and Y. H. Yang, "Privacy intensity policy for optimizing source node location in wireless sensor networks," Laser \& Optoelectronics Progress, vol. 57, no. 24, Article ID 241017, 2020.

[16] A. Pang, F. Chao, H. Zhou, and J. Zhang, "The method of data collection based on multiple mobile nodes for wireless sensor network," IEEE Access, vol. 8, pp. 14704-14713, 2020.

[17] Y. Cheng, Q. Liu, J. Wang, S. Wan, and T. Umer, "Distributed fault detection for wireless sensor networks based on support vector regression," Wireless Communications and Mobile Computing, vol. 2018, Article ID 4349795, 8 pages, 2018.

[18] Y. Tang and Y. Dai, "Wireless strain synchronization acquisition method based on kalman filter," Journal of Physics: Conference Series, vol. 1754, no. 1, Article ID 012064, 2021.

[19] F. Shi, "Simulation of information segment fusion point extraction method for wireless sensor network," Computer Simulation, vol. 35, no. 4, pp. 262-265, 2018.

[20] X. Yu, Y. Chu, F. Jiang, Y. Guo, and D. Gong, "SVMs classification based two-side cross domain collaborative filtering by inferring intrinsic user and item features," KnowledgeBased Systems, vol. 141, pp. 80-91, 2018.

[21] H. Khan, M. A. Jan, M. Alam, and W. Dghais, "A channel borrowing approach for cluster-based hierarchical wireless sensor networks," Mobile Networks and Applications, vol. 24, no. 4, pp. 1306-1316, 2019.

[22] X. Yu, F. Jiang, J. Du, and D. Gong, "A cross-domain collaborative filtering algorithm with expanding user and item features via the latent factor space of auxiliary domains," Pattern Recognition, vol. 94, pp. 96-109, 2019. 